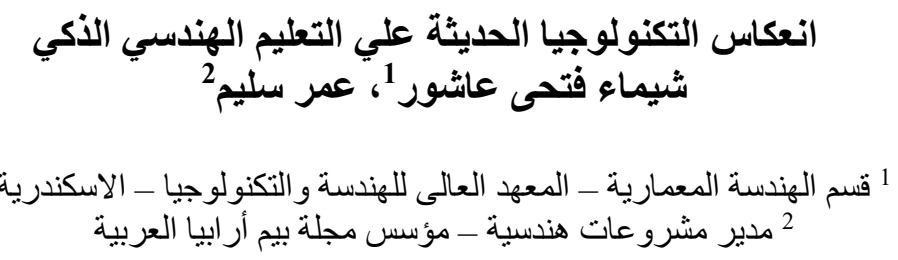

ملخص البحث

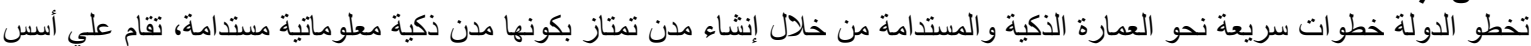

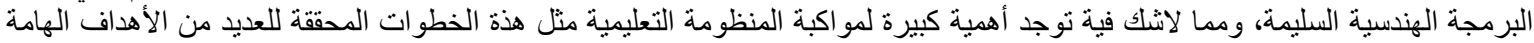

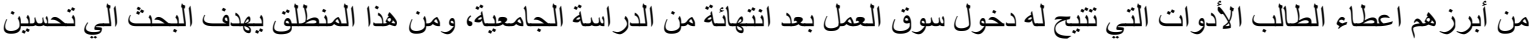

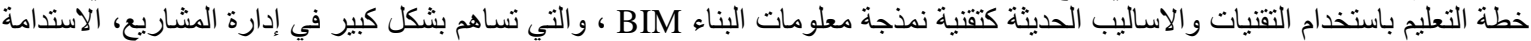

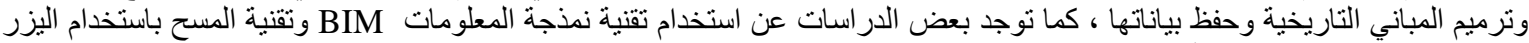

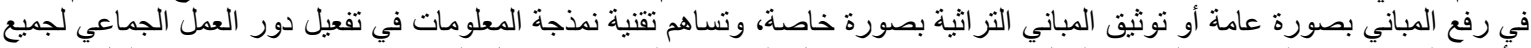

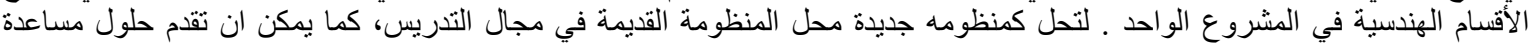

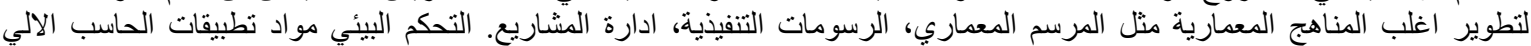

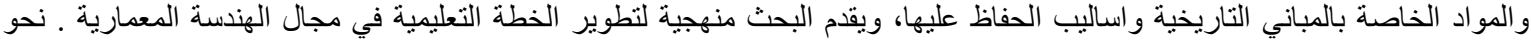

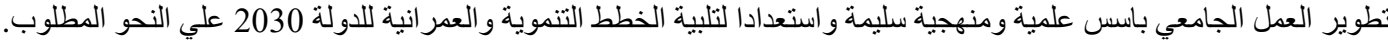

الكلمات المفتاحية: تقنية نمذجة معلومات البناء ، التعليم الذكي ، التصميم المتكامل الاستدامة، صناعة البناء ، المهارات الطلابية.

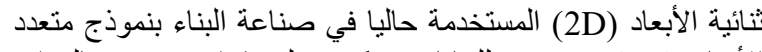

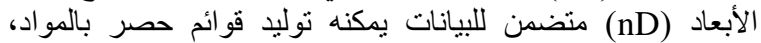

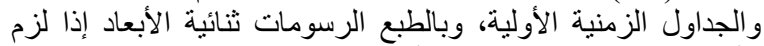

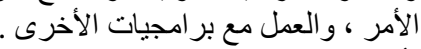

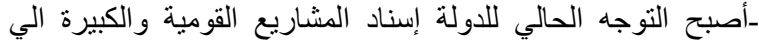

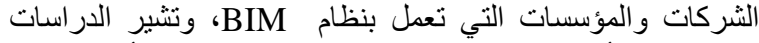

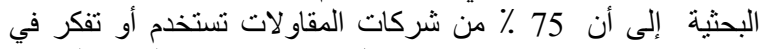
استخدام BIM في شركاتها ويفضل توظيف من يمتلكون المعرفة

بمهار ات هذه التقنية.

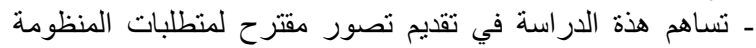

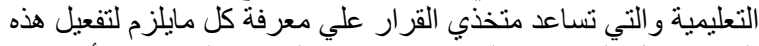

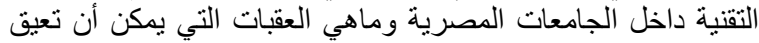

تنفيذه.

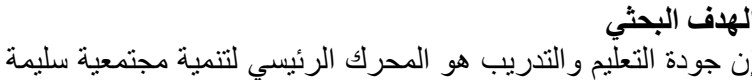

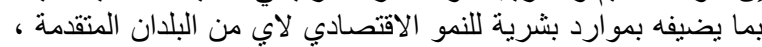

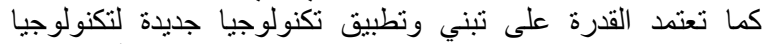

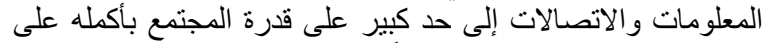

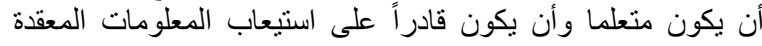

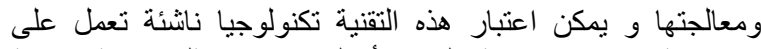

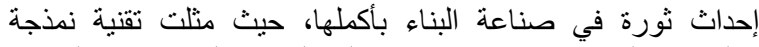

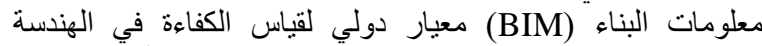
المعمارية والبناء (AEC) ومجمو عة من خدمات البناء الأخرى.

1. تعريف تقتية نمذجة معلومات البناء BIM التهنة

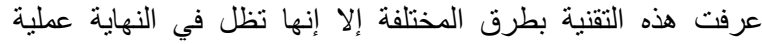

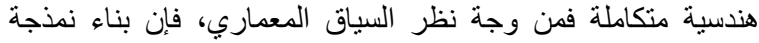
المعلومات (BIM) هو منهجية تصميم تمكن جميع تفاصيل التصميم والمقررات
تعتبر تقنية نمذجة معلومات البناء

(Building Information Management, BIM) واحدة من أهم التطورات الواعدة الأخيرة في مجالات الهندسة

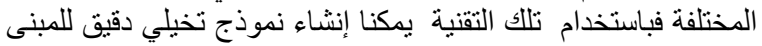

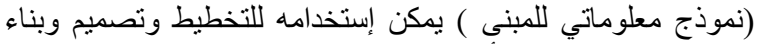

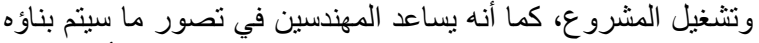

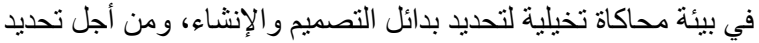

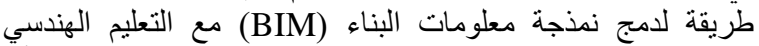

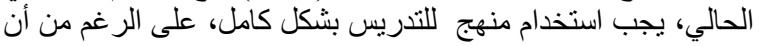

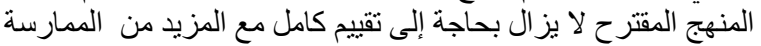

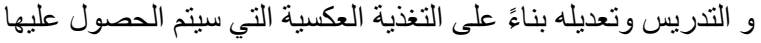

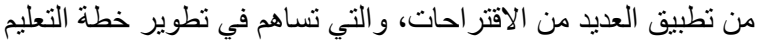

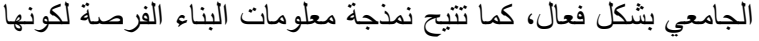

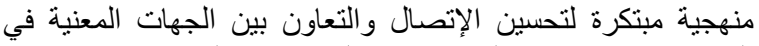

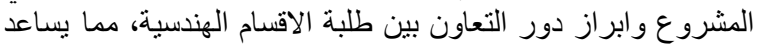

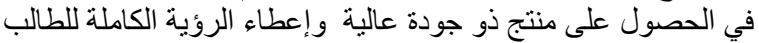

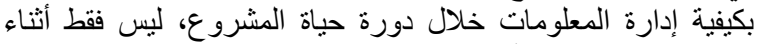
التصميم و البناء ولكن أثناء التشغيل و الصيانة.

أهمية الدراسة

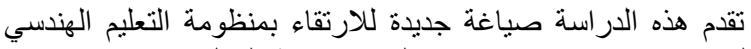

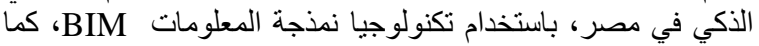

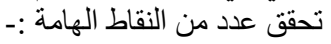

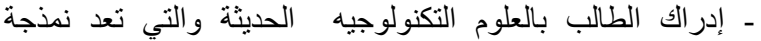

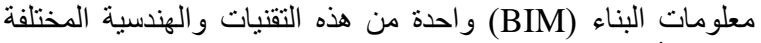
باعتبار أن الدراسة الجامعة فرصة واهدة للتعرف على هذه هذه التقنيات التي تستخدم في صناعة البناء و التشييد.

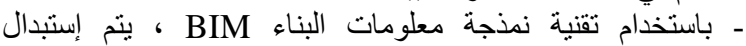
الرسومات 


\section{Problem Solving Method}

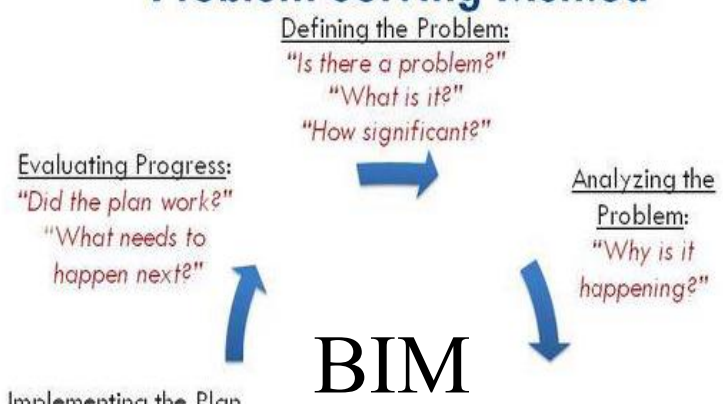

Implementing the Plan

$$
\text { with Fidelity: }
$$

"Are you doing what you said you would do? How do you know?"

$\sqrt{15}$
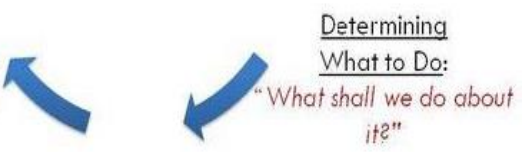

شكل (2) طرق حل المشكلات ودعم اتخاذ القرار السليم (4)

يوضح الثكل السابق أهمية التقنية الذكية ودمجها في أسس التعليم لما

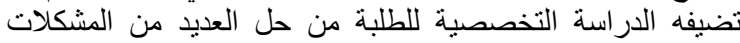
التصميمية التي تواجه المهندس في تحليل المشروع وتحسين جودته وأهمها : التعريف بالمشكلة التصميمية وموقع حدوثها حيث توفر برمجيات

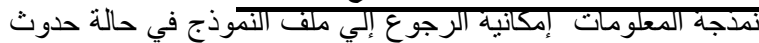

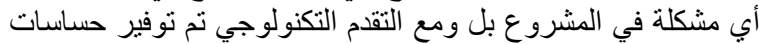

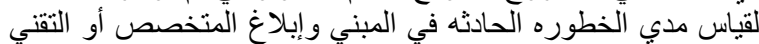

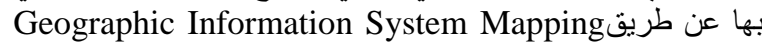
GSI تحليل المشكلة لحل أي مشكله تصميمية وإتخاذ القرارات السليمه

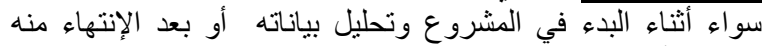

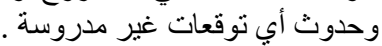

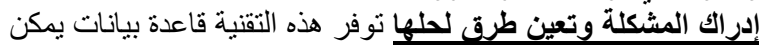

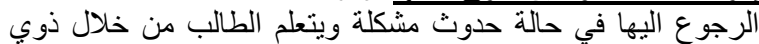

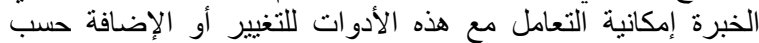

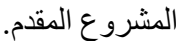

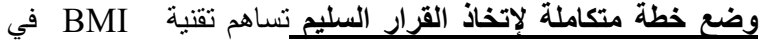

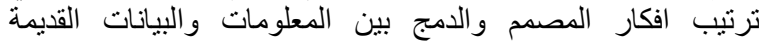

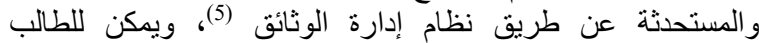

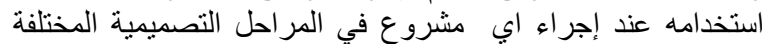
للتدرب علي الإستخدام ضمن برمجيات التقنية المستخدمة.

1.3 تقييم العملية التصميمية والاجراءات المتبعة لحل المثكلة:

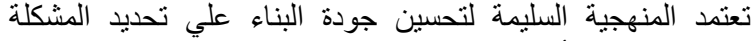
والمؤثرات التي أدت إلي ذلك وتنعكس هذه المرحلة إيجابيا علي الئي

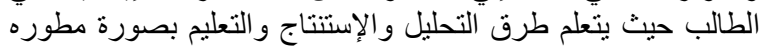

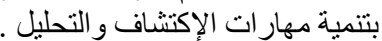

1.1.3 إنعكاس تطبيق البيم في الجامعات على سوق العمل العبل

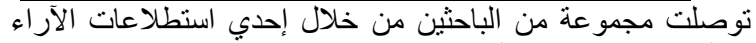

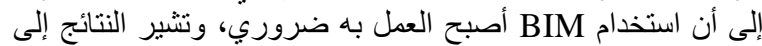

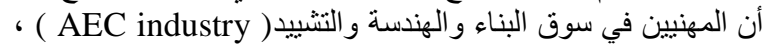

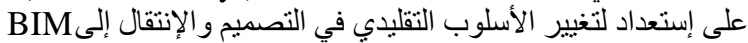

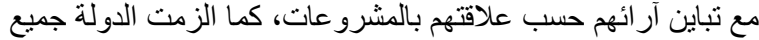
الثركات والمؤسسات التي تعمل في المدن الذكية الجديدة باستخدام
و الخصائص من أن يتم عقدها ضمن حزمة تعاونية رقمية ومعلوماتية.

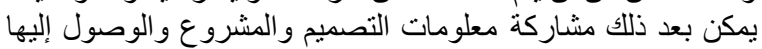

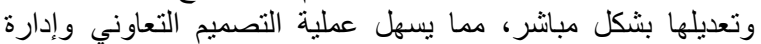

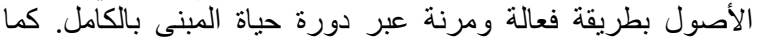

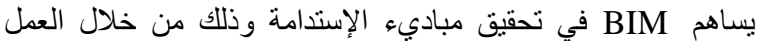

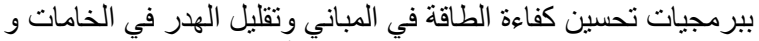

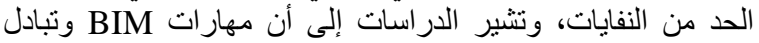

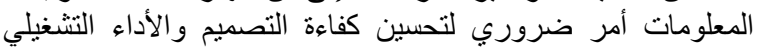

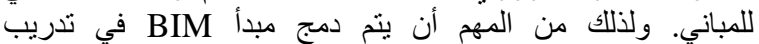

المتخصصين في البناء في المستقبل.

$$
1.1
$$

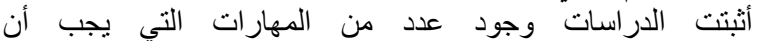

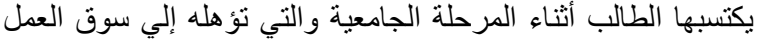

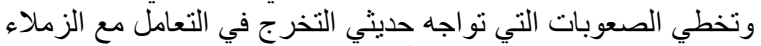

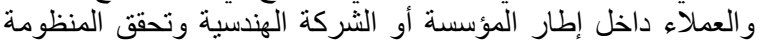

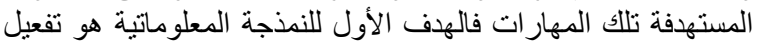
دور التعاون بين التخصصات الهندسية المختلفة وحددت تلإلك المهار التهات

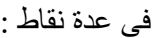
ـ ـالحاجة إلى التفكير الناقد وطرق حل المشكلات التصميمية. ـالتعاون عبر الثبكات والريادة من خلال التأثير. ـالقدرة على التكيف مع العمل الجماعي. ـ المبادرة وتقديم الفكر المبتكر و الواعي.

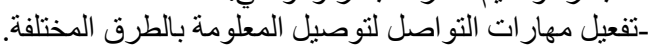
ــإقرق الوصول إلي المعلومات وتحليلها. - إتاحة الفرصة للخيال و الإبداع التقني.

كما حددت الجودة التعليمية 4 مهار آت يجب التهاب أن يكتسبها الطالب أثناء

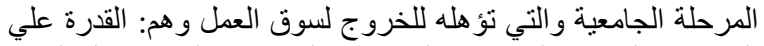

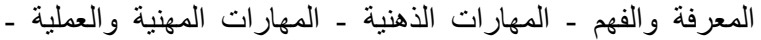
المهار ات العامة و المنقوله. ومن ثم يتم دمج جميع المهار ات المات السابقة ومطابقتها بما يمكن أن يقدمة تقنية نمذجة معلومات البناء للخريج الجامعي حين ذلك.

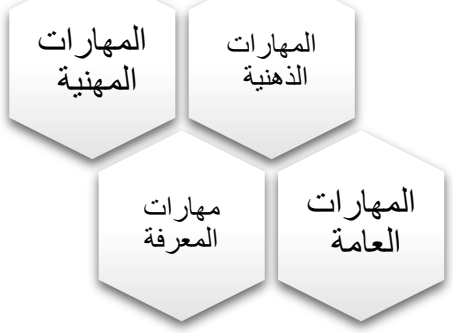

شكل (1) المهار ات التعليمية لتأهيل الطالب الجامعي لسوق العمل

1.2 بناء نمذجة المعلومات ودعم اتخاذ القرار

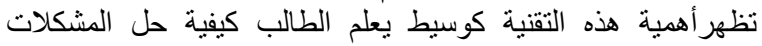

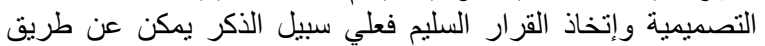

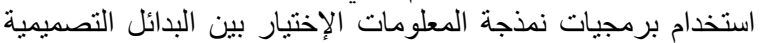

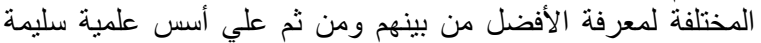

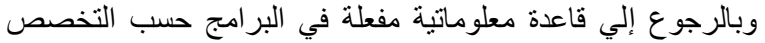

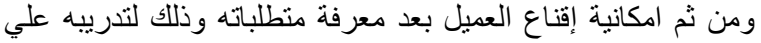
مواجهة سوق العمل، فباستغلال تللك الموارد (العقول المبتكرة ) التي التي

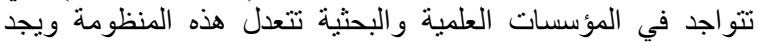
العميل مطلبه مستوفيا الإشتر اطات البنائية و البيئية . 
وفيما يلي جدول (1) يحتوي علي بر امج ماجستير إدارة الأعمال ذات

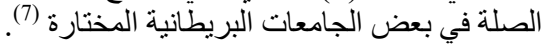

و لإستيعاب أفضل لتلك التقنية يحتاج الطالب فهما من المرحلة الجامعية

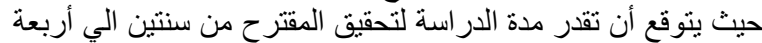

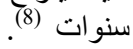

جدول (1) بر امج ماجستير إدارة الأعمال و أهميه در استها

\begin{tabular}{|c|c|}
\hline المميزات & البرنـامج \\
\hline 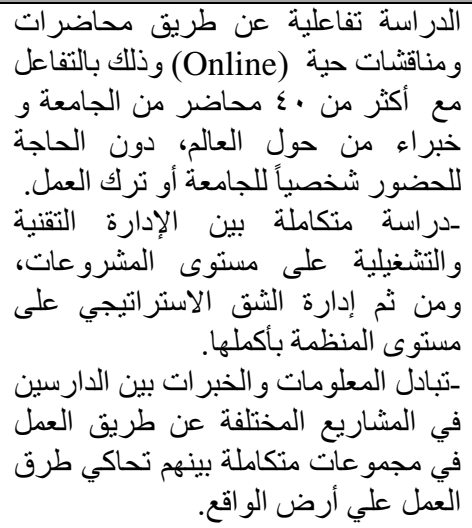 & $\begin{array}{c}\text { Middlesex } \\
\text { معلتير إدارة نمذجة البناء - لندات - لنداء) }\end{array}$ \\
\hline 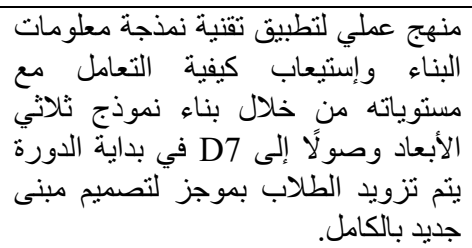 & 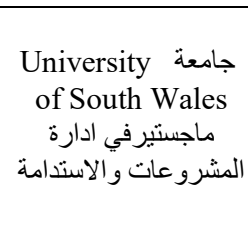 \\
\hline 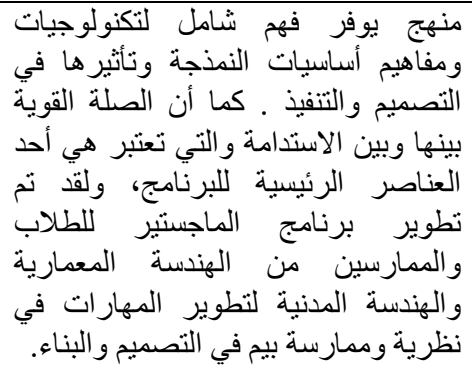 & 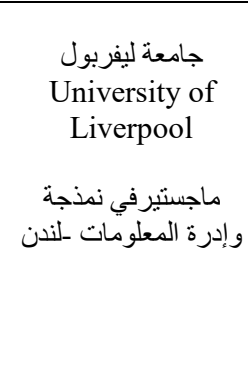 \\
\hline 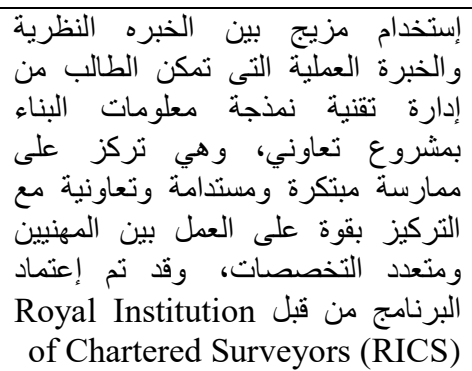 & 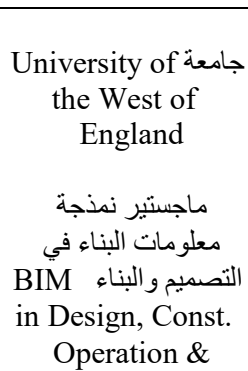 \\
\hline
\end{tabular}

تقنية نمذجة معلومات البناء في جميع مبانيها بداية من البنية التحنية للمدينة . ويضاف علي ذلك بعض المباني المنشأه حديثا كالمتحف المصري

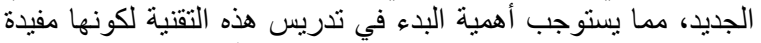

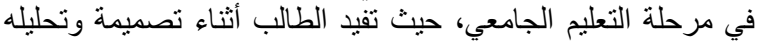

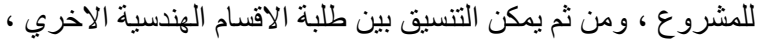

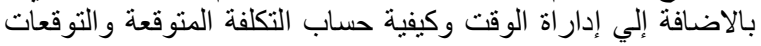

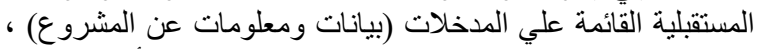

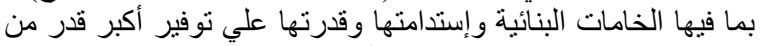

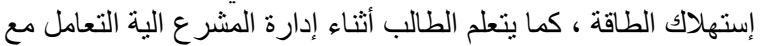

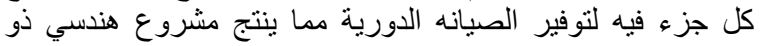
جودة عالية وباقل نسبة خطورة ممكنة.

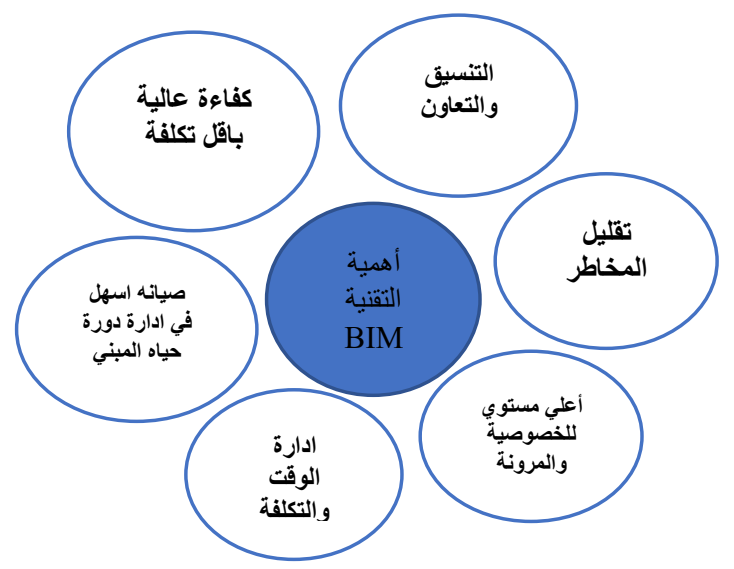

شكل (3 ) فو ائد استخدام نمذجة المعلومات في التصميم

2.1.3 نماذج تطبيقية لبرنامج تدريس البيم بالجامعات

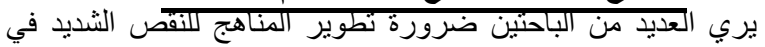

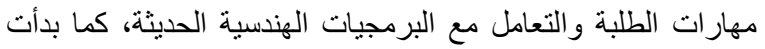
بعض الجامعات في تدريس BIM وكثير من الباحثين وذوي الخبرة

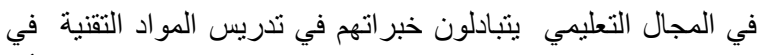

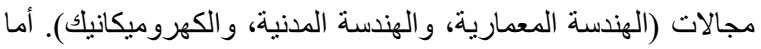

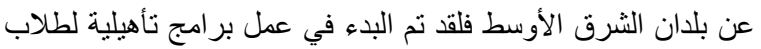
الماجستير و الدكتور اه في تخصص الأنس نمذجة البناء. (6)

إنطلقت بعض الجامعات المصرية لتدريس تقنية نمذجة المعلومات في

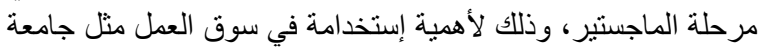

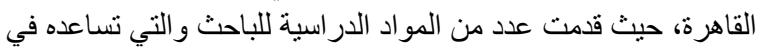

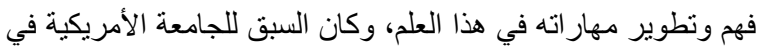

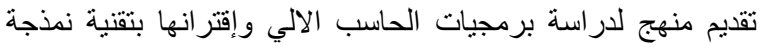

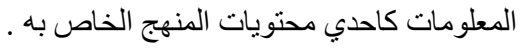

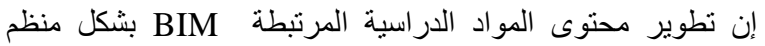

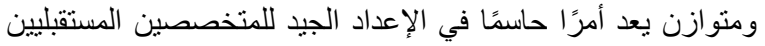

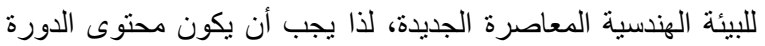

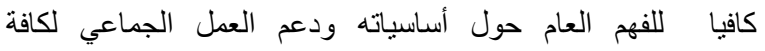
التخصصات الهندسية لتقديم محتوي متكامل . 
ـ ـ تحقق تقنية تكنولوجيا المعلومات مبدألتعاون حين دمجها داخل

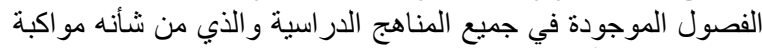
سوق العمل وتأهيل ألطالب الجامعي علي المرحلة المقبلة الكبلة في حياته مراكية العملية والتي تستهدفها المنظومة التعليمية باكملها.

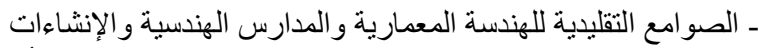

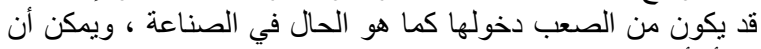

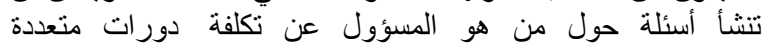
التخصصات.

\section{}

لتحقيق المنظومة التعليمية المطلوبة يجب وضع رؤية محددة هدفها

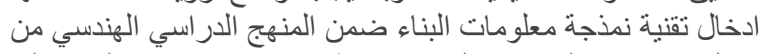

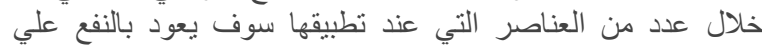

الطالب الجامعي موضحة في جدول (3).

من المتوقع حدوث طفره في مجال التعليم المعماري كعاذد لاستخدام تلك الكنظومة ومن أهم النقاط المنوقعة :-

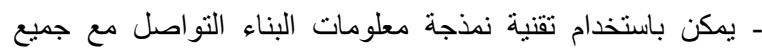

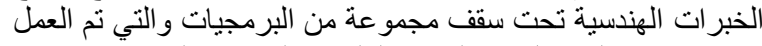

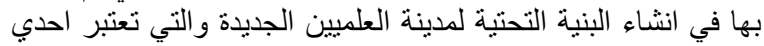

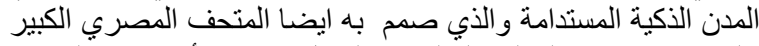
بالقاهرة بعد هذه العملية التعليمية بالكامل ، يجب الني أن يكون الطلاب النياب قادرين على:

1- - إدراك أهمية تقنية معلومات البناء و والفوائد المحتملة التي

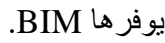

2- فهم مصطلحات عملية النمذجة وأبعادها وقدرتها على تلبية احتياجات صناعة البناء و التشييد.

3- التعرف على نطاق أنشطة البناء للفكر التكنولوجي الحديث

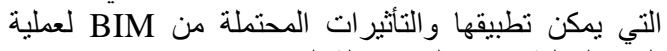
البناء الحالية فيما يتعلق بجدولة المشرو ع ومر اقبتها.

4- - حدد البرامج والأجزةة المناسبة للعمل باستخدام BIM

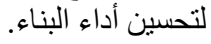

5- تحديد إتجاهات الدراسة المستقبلية للعمل بفكر الندجة

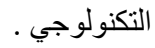

6- أن تتلاقي الإفكار باستخدام شبكات الانترنت لتعلم الطلبة مبدأ

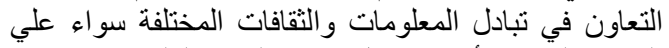
الصعيد الهندسي أو اي مجال يخص البحثث العلمي.
4. مراحل تطوير الطالب أثناء الاراسة الجامعية

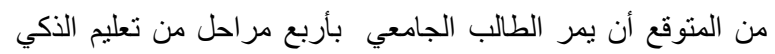
و التقني ليصبح مهندس نموذجي:

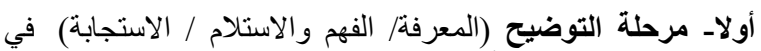

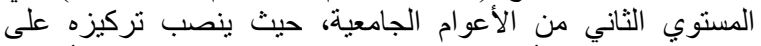

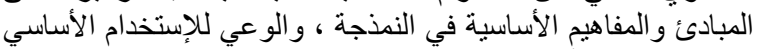
لتقنيات MIB الأساسية وتقدير قضايا التعاون و وقابلية التشغيل البيني .

ثانيا- مرحلة العمل (الفهم / التطبيق والاستجابة/ التقيبم) المستوي

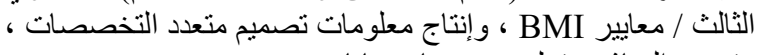
وتتسيق النماذج وتوليد مجمو عات بيانات .

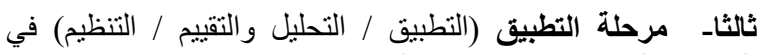

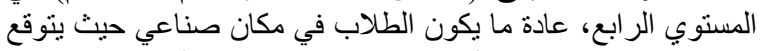

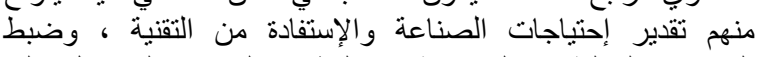

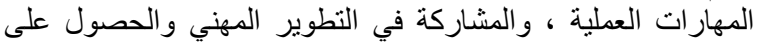

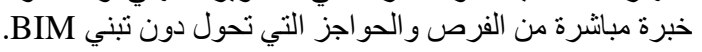

- مرحلة التعاون ( التقييم و التوصيف) المستوي الخامس بنطبيق

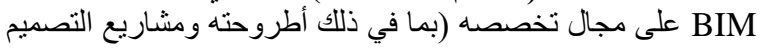

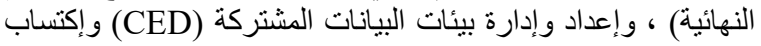

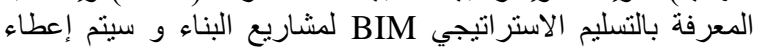
الطلاب مشاكل حقيقية لحلها.

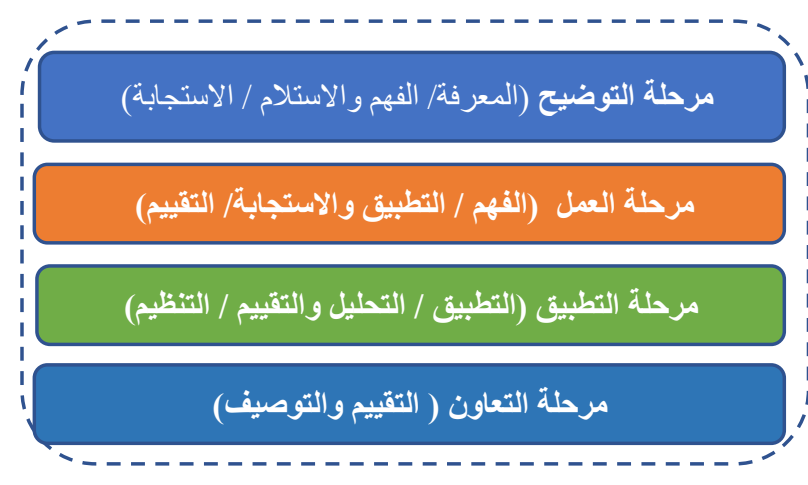

$$
\text { شكل(4) المر احل المختلفة من التعليم الجامعي الذكي }
$$

5. 5. التحديات والصعوبات المتوقعة

من المرجح أن تواجه عملية التغيير مقاومة من النظام واللوائح

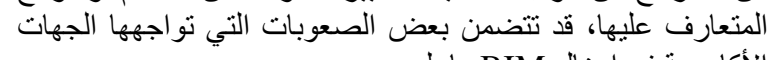

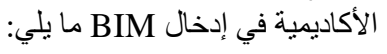

ـ أسئلة حول كيفية إضافة مو اضيع جديدة في المناهج المزدحمة. ـ تغيير عادات التنديس التي تأسست على مدى سنوات عديدة.

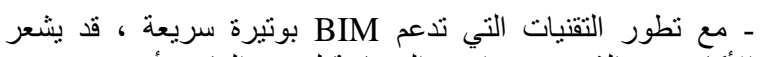

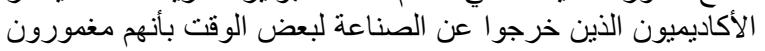

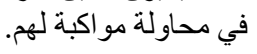




$$
\text { شيماء فتحي عاثور محح، عصر سليم " إنعكاس التكنولوجيا الحلثية علي التعليم الهندسي الذكي " }
$$

جدول (3) العناصر المقترحة والعائد المتوقع لتدريس تقنية معلومات

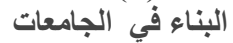

\begin{tabular}{|c|c|c|}
\hline العائد المتوقع & محتوي المواد الدراسية & عناصر المقترح \\
\hline ـ البناء يمكن باستخدام تقتية نمذجة معلومات & 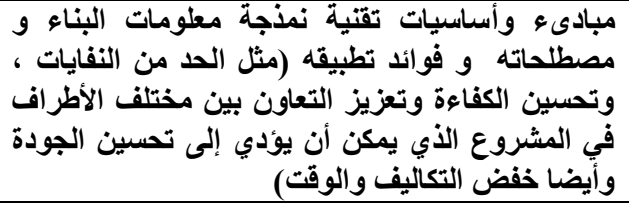 & مبلومات البناء وأساسيات تقتية نمذجة \\
\hline 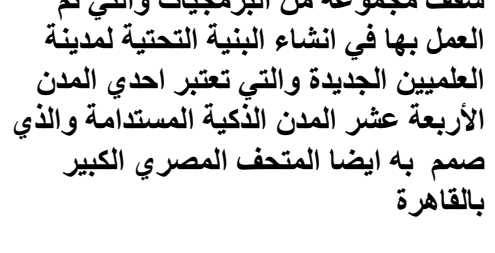 & 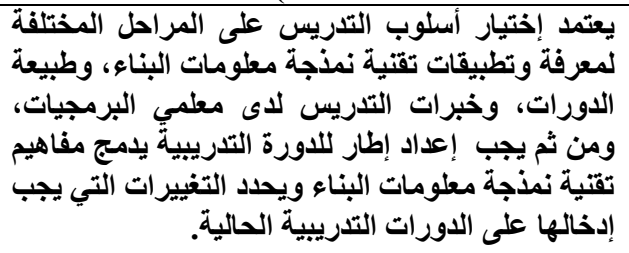 & إختيار أساليب تدريس مختلفة \\
\hline 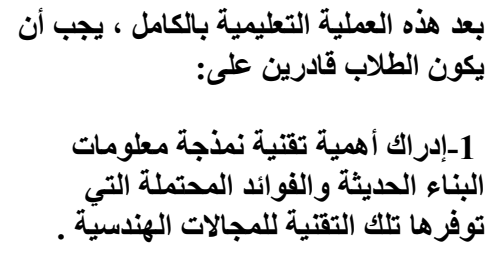 & 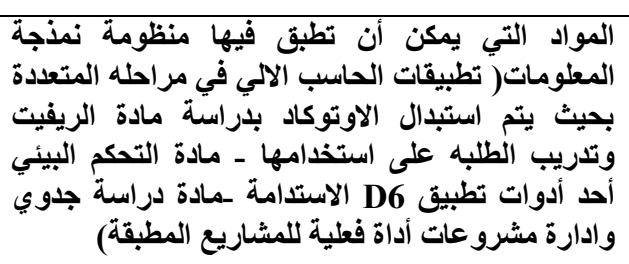 & منظومة نمذجبة المعلومات أن ت تطبق فيها \\
\hline 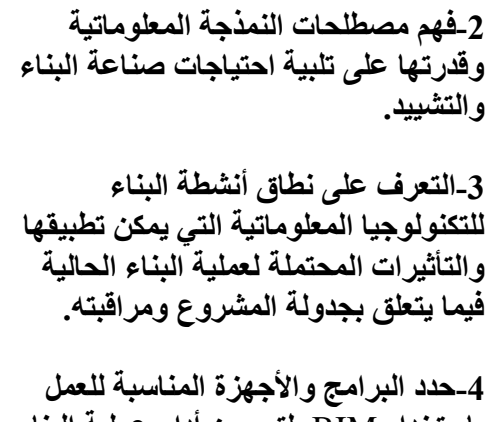 & 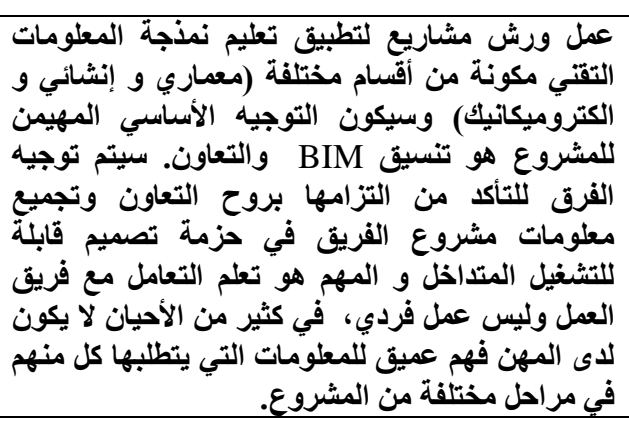 & نمذجة معلومات البناء المشتركة لتطبيق تقتية \\
\hline 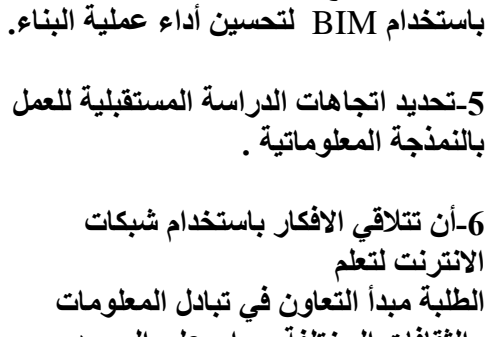 & 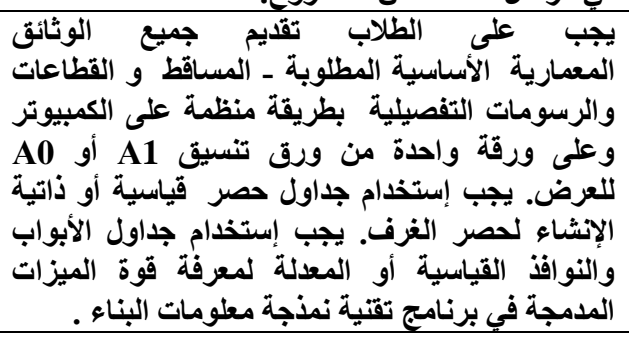 & سوق العمل تقلم المشاريع مماثلة لمتطلبات \\
\hline الهندسي أو اي مجال يخص المقاء البحث الصعيد العلمي. & 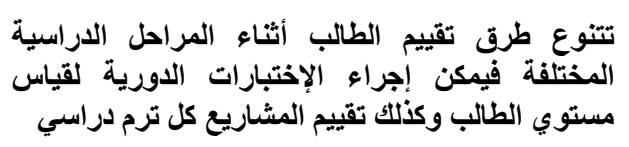 & وفعالية أساليب التقيبيم لتعلم الطلاب \\
\hline
\end{tabular}

تصعي المنارات التعليمة المكتسبة اثناء المر احل التعليمية

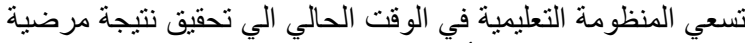

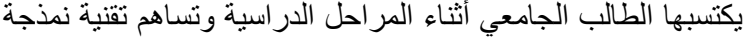

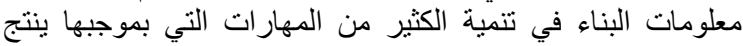

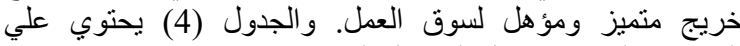
المهار ات المكتسبة خلال المر احل الدر اسية . 


\begin{tabular}{|c|c|c|c|}
\hline المهارة التعليمية المكتسبة من خلال & المرحلة الاراسية & تنمية المهارات الطلابية & التربوية \\
\hline \multirow[t]{2}{*}{ 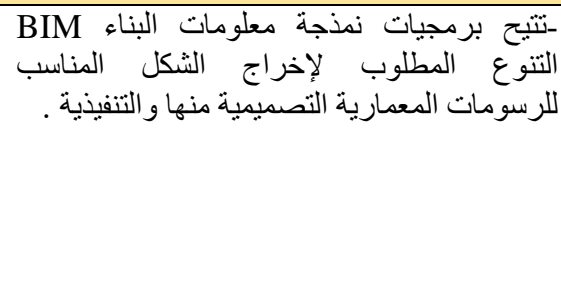 } & 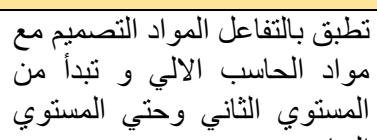 & القفيمرية المشروعالهات و ومستوياتهات & \multirow{5}{*}{ 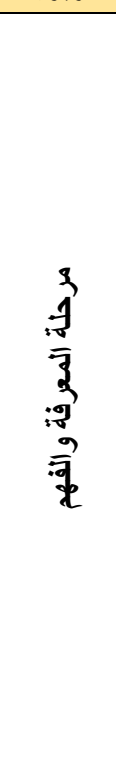 } \\
\hline & 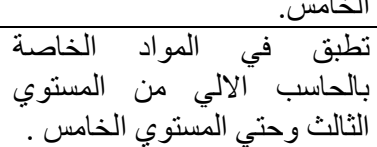 & الأبعاد الرسومات و النماذج ثلاثية & \\
\hline \multirow[t]{2}{*}{ 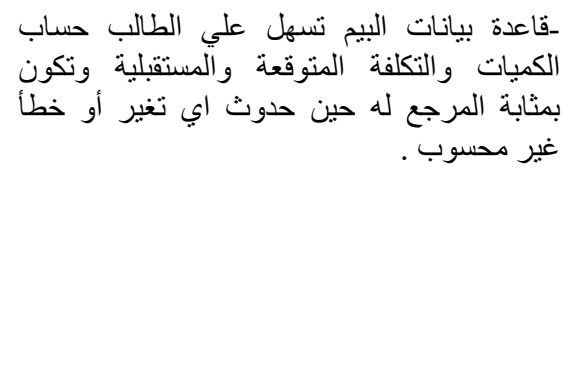 } & 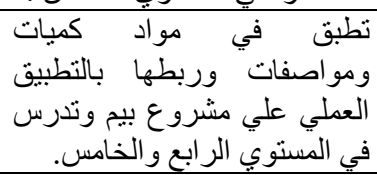 & 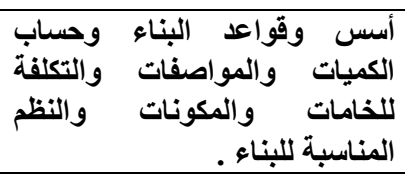 & \\
\hline & 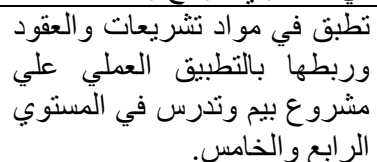 & 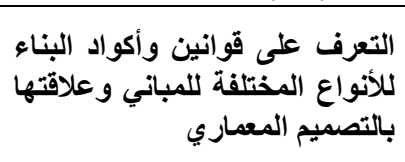 & \\
\hline \multirow{2}{*}{ 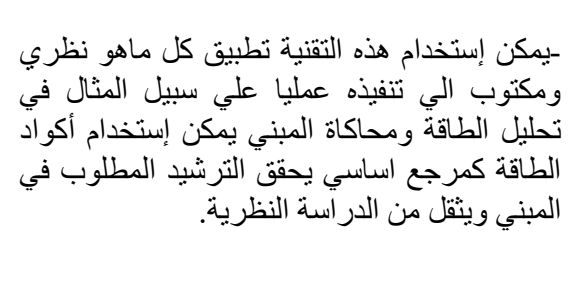 } & 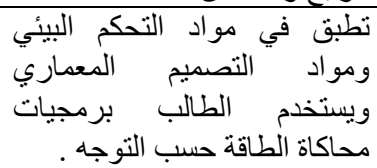 & 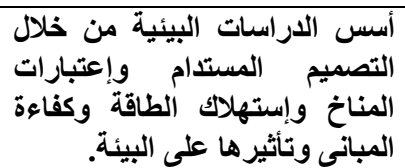 & \\
\hline & 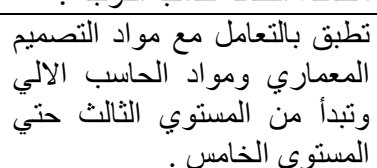 & 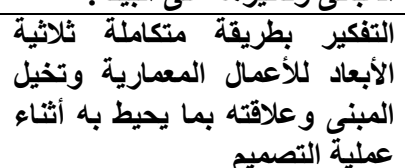 & \multirow{4}{*}{ 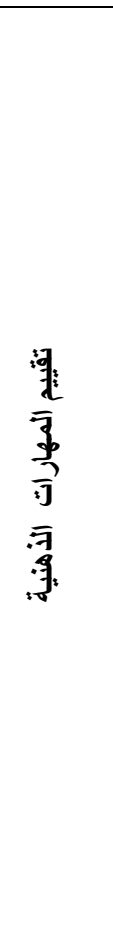 } \\
\hline \multirow[t]{2}{*}{ 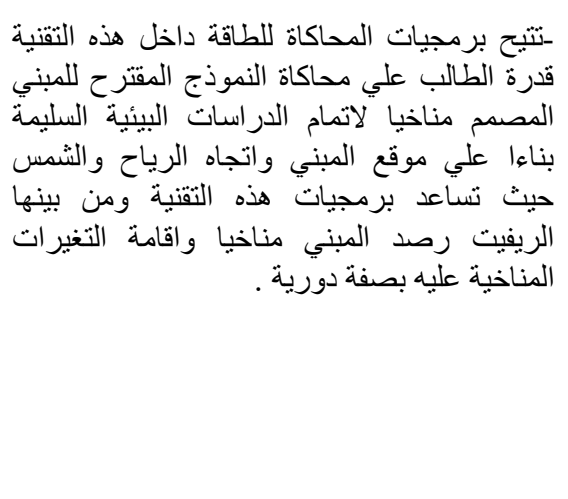 } & 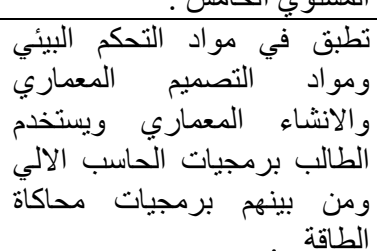 & 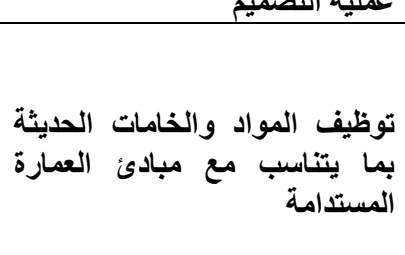 & \\
\hline & 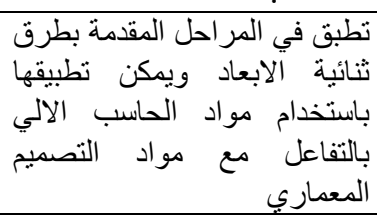 & 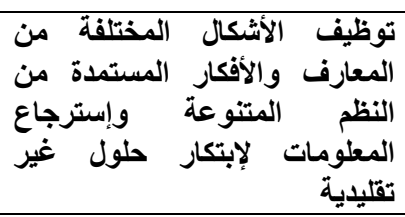 & \\
\hline 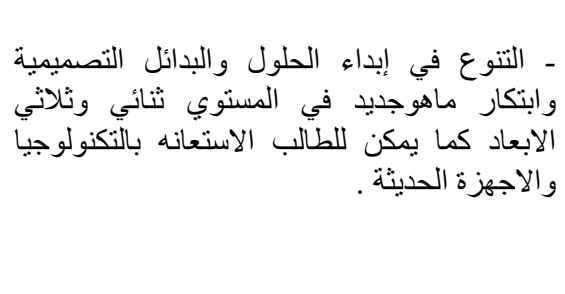 & 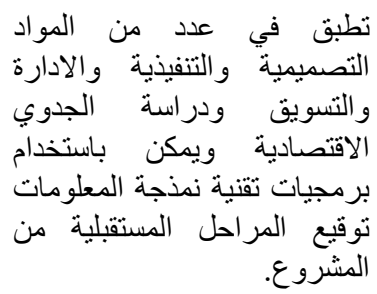 & 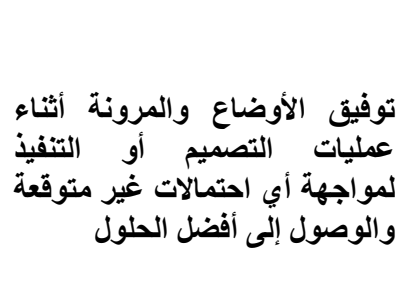 & \\
\hline
\end{tabular}


شيماء فتحي عاثشور محلم، عر سليم " إنعكاس التكنولوجيا الحسيثة علي التعليم الهندسي النكي "

جدول (4) تابع المهارات التعليمية المكتسبة لتقتية نمذجة المعلومات خلال المراحل الدراسية الجامعية

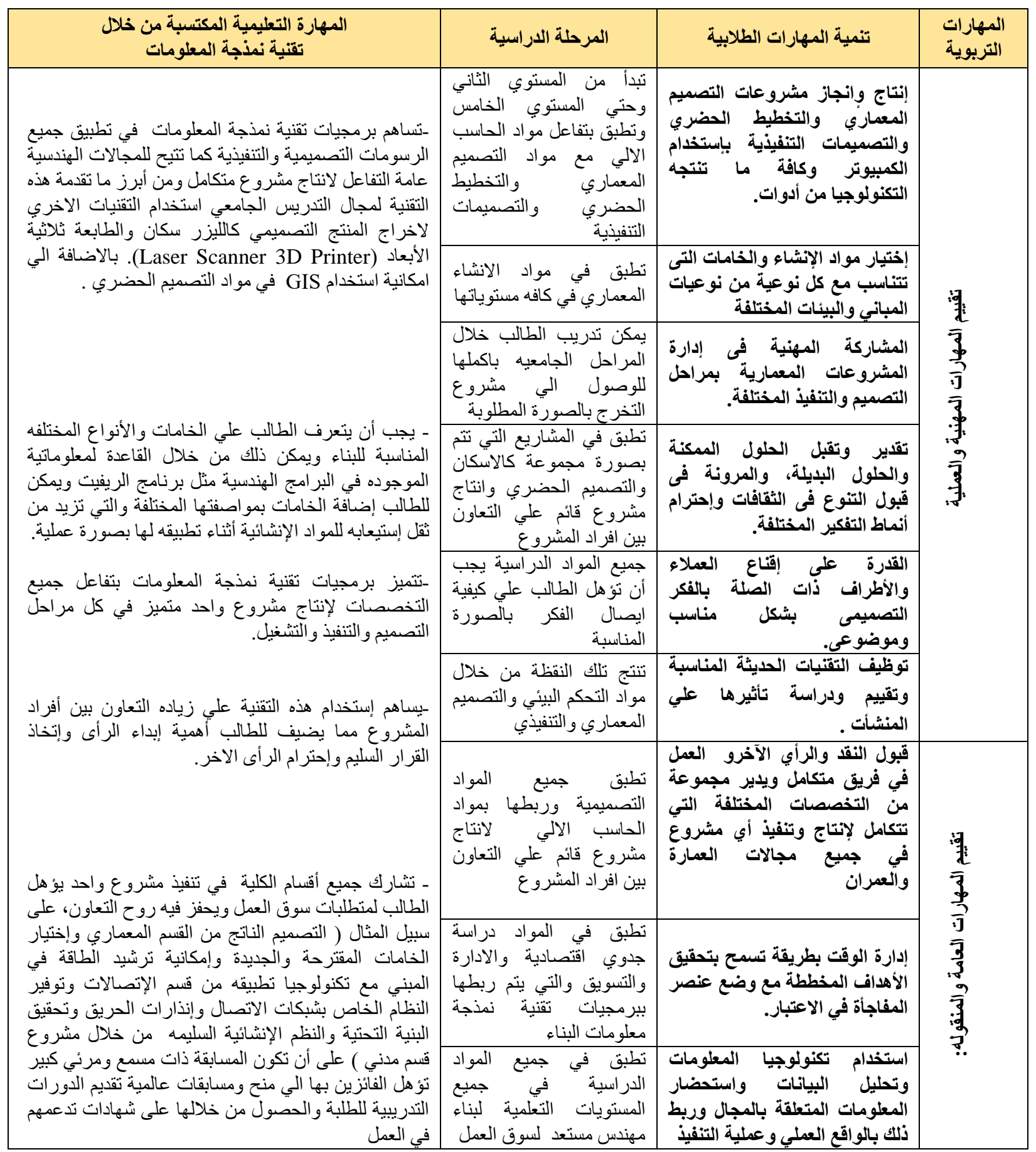


1- Stavric, M., et al., From 3D building information modeling towards 5D city information modeling. 3D Issues in Urban and Environmental Systems.

2- Bologna, Italy: Società editrice ESCULAPIO, COST, 2012.

3- Barison, M.B. and Santos, E.T., 2010, June. BIM teaching strategies: an overview of the current approaches. In Proc., ICCCBE 2010 international conference on computing in civil and building engineering.

4- Sacks, R. and Barak, R., 2010. Teaching building information modeling as an integral part of freshman year civil engineering education. Journal of professional issues in engineering education and practice, 136(1), pp.30-38. Sacks, R. and Pikas, E., 2013.

5- Van Nederveen, G. and F. Tolman, Modelling multiple views on buildings. Automation in Construction, 1992. 1(3): p. 215-224.

6- Building information modeling education for construction engineering and management. I: Industry requirements, state of the art, and gap analysis. Journal of Construction Engineering and Management, 139(11), p.04013016.

7- Adamu, Z. and Thorpe, T., 2016. How universities are teaching bim: A review and case study from the UK. Journal of Information Technologyin Construction, 21, pp.119-139

8- Clevenger, C.M., Ozbek, M., Glick, S. and Porter, D., 2010, February. Integrating BIM into construction management education. In EcoBuild Proceedings of the BIM-Related Academic Workshop.

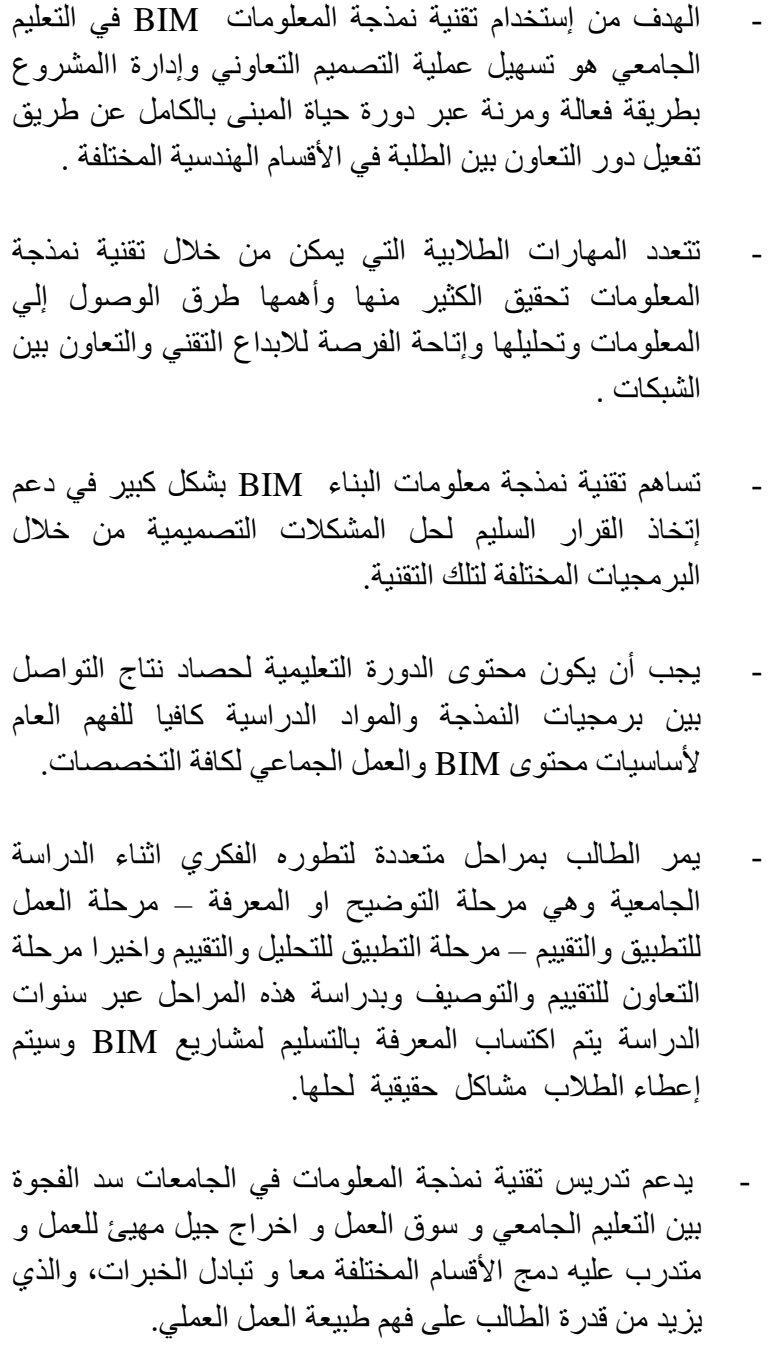

